\title{
RESULTS ON WEIGHTED NORM INEQUALITIES FOR MULTIPLIERS

\author{
BY
}

\author{
DOUGLAS S. KURTZ AND RICHARD L. WHEEDEN ${ }^{1}$
}

\begin{abstract}
Weighted $L^{p}$-norm inequalities are derived for multiplier operators on Euclidean space. The multipliers are assumed to satisfy conditions of the Hörmander-Mikhlin type, and the weight functions are generally required to satisfy conditions more restrictive than $A_{p}$ which depend on the degree of differentiability of the multiplier. For weights which are powers of $|x|$, sharp results are obtained which indicate such restrictions are necessary. The method of proof is based on the function $f^{\sharp}$ of $\mathbf{C}$. Fefferman and $\mathbf{E}$. Stein rather than on Littlewood-Paley theory. The method also yields results for singular integral operators.
\end{abstract}

1. Let $m(x)$ be a bounded function on $\mathbf{R}^{n}$ and consider the multiplier operator $T f$ defined initially for functions $f$ in the Schwartz space $\mathcal{S}$ by $(T f)^{\wedge}(x)=m(x) \hat{f}(x)$, where $\hat{g}$ is the Fourier transform of $g$. Denote by $s$ a real number greater than or equal to $1, l$ a positive integer, and $\alpha=\left(\alpha_{1}, \ldots, \alpha_{n}\right)$ a multi-index of nonnegative integers $\alpha_{j}$ with length $|\alpha|=\alpha_{1}+\cdots+\alpha_{n}$. We say $m \in M(s, l)$ if

$$
\sup _{R>0}\left(R^{s|\alpha|-n} \int_{R<|x|<2 R}\left|D^{\alpha} m(x)\right|^{s} d x\right)^{1 / s}<+\infty \quad \text { for all }|\alpha|<l .
$$

The condition (1.1) has been known to be related to multipler theorems for some time. The classic works in this direction are the theorems of Marcinkiewicz (see [18]) and Hörmander-Mikhlin (see [7]):

Theorem A. Let $n=1,1<p<\infty$, and $m \in M(1,1)$. Then there exists a constant $C$, independent of $f$, such that $\|T f\|_{p}<C\|f\|_{p}$.

TheOREM B. Let $l>n / 2,1<p<\infty$, and $m \in M(2, l)$. Then there exists $a$ constant $C$, independent of $f$, such that $\|T f\|_{p}<C\|f\|_{p}$.

Much work has been done to extend these results. Using interpolation methods, Calderón and Torchinsky [2] have considered the condition $m \in$ $M(s, l)$ for $s \geqslant 2$ and $l>n / s$. Hirschman [6], Krée [11], and Triebel [20] have extended these results in various directions to weighted $L^{p}$ spaces for weights which are powers of $|x|$. More recently, Kurtz [12] extended Theo-

Received by the editors November 9, 1978.

AMS (MOS) subject classifications (1970). Primary $42 \mathrm{~A} 18$.

${ }^{1}$ Supported in part by NSF Grant MCS 77-03980. 
rems $\mathrm{A}$ and $\mathrm{B}$ to $L^{p}$ spaces with more general weights by using the weighted norm inequalities derived in [15] for the function $g_{\lambda}^{*}$.

The purpose of this paper is two-fold. We consider $s<2$ and present a method of proof based on the function $f^{\sharp}$ of Fefferman and Stein [5] rather than on Littlewood-Paley theory.

We say $f \in L_{w}^{p}\left(\mathbf{R}^{n}\right), 1<p<\infty$ and $w(x)>0$, if

$$
\|f\|_{p, w}=\left(\int_{\mathbf{R}^{n}}|f(x)|^{p} w(x) d x\right)^{1 / p}<+\infty .
$$

The weights $w$ we will consider satisfy an $A_{r}$ condition; i.e., $w \in A_{r}$ if there is a constant $C$ such that

$$
\begin{gathered}
\left(\frac{1}{|Q|} \int_{Q} w(x) d x\right)\left(\frac{1}{|Q|} \int_{Q} w(x)^{-1 /(r-1)} d x\right)^{r-1}<C, \quad 1<r<\infty, \\
\frac{1}{|Q|} \int_{Q} w(x) d x<C \underset{Q}{\operatorname{ess} \inf w, \quad r=1,}
\end{gathered}
$$

for all cubes $Q \subset \mathbf{R}^{n}$. When $r=1$, the condition that $w \in A_{1}$ means $w^{*}(x)<$ $C w(x)$ for almost every $x$, where $g^{*}$ is the Hardy-Littlewood maximal function of $g$. Finally, $w \in A_{\infty}$ if there exist positive constants $C$ and $\delta$ such that for any cube $Q \subset \mathbf{R}^{n}$ and for any measurable set $E \subset Q$,

$$
\frac{m_{w}(E)}{m_{w}(Q)}<C\left(\frac{|E|}{|Q|}\right)^{\delta},
$$

where $m_{w}(E)=\int_{E} w(x) d x$. Results concerning $A_{p}$ functions can be found in Muckenhoupt [13] and Coifman and Fefferman [3]. Note, in particular, that $w \in A_{p}$ implies $w \in A_{\infty}$.

We use $p^{\prime}$ to denote the index conjugate to $p: 1 / p+1 / p^{\prime}=1, p>1$.

The main result of this paper is:

THEOREM 1. Let $1<s<2, n / s<l<n$, and $m \in M(s, l)$. If

(1) $n / l<p<\infty$ and $w \in A_{p l / n}$, or

(2) $1<p<(n / l)^{\prime}$ and $w^{-1 /(p-1)} \in A_{p^{\prime} l / n}$,

then there is a constant $C$, independent of $f$, such that

$$
\|T f\|_{p, w}<C\|f\|_{p, w} .
$$

When $l<n$, we may take $p=n / l$ in (1) and $p=(n / l)^{\prime}$ in (2). If

(3) $w^{n / l} \in A_{1}$,

there is a constant $C$, independent of $f$ and $\lambda$, such that

$$
m_{w}\left(\left\{x \in \mathbf{R}^{n}:|T f(x)|>\lambda\right\}\right)<\frac{C}{\lambda}\|f\|_{1, w}, \quad \lambda>0 .
$$

Using interpolation, other conditions on the weight can be found which guarantee that $T$ is a bounded operator. One result which we will prove is: 
THEOREM 2. If $1<p<\infty, 1<s<2, n / s<l<n$, $m \in M(s, l)$, and $w^{n / l} \in A_{p}$ then

$$
\|T f\|_{p, w}<C\|f\|_{p, w}
$$

for a constant independent of $f$.

This result does not give the best possible condition on the weight. When $w(x)=|x|^{\beta}$, we have $w \in A_{p}$ if $-n<\beta<n(p-1)$. Interpreting Theorem 1 for such $w$ and using interpolation with change of measures, we will show:

TheOrem 3. Let $1<s<2, n / s<l<n$, and $m \in M(s, l)$. If $1<p<\infty$ and $\max \{-n,-l p\}<\beta<\min \{n(p-1), l p\}$, then there is a constant $C$, independent of $f$, such that

$$
\|T f\|_{p,|x|^{\beta}}<C\|f\|_{p,|x|^{\beta}} .
$$

In particular, if $n / l<p<(n / l)^{\prime}$, we get $-n<\beta<n(p-1)$; we may also take $p=n / l$ and $p=(n / l)^{\prime}$ if $l<n$.

We will show that this result is sharp with the possible exception of the endpoint values of $\beta$.

Let $\check{g}$ denote the inverse Fourier transform of $g$. If we set $K=\check{m}$, then for $f \in \mathcal{S}, T f(x)=(K * f)(x)$. Our proof of Theorem 1 is based on using information about $m$ to get estimates on approximations to $K$, so it is not surprising that the technique carries over to convolution operators.

Denote by $\Sigma=\Sigma_{n-1}=\left\{x \in \mathbf{R}^{n}:|x|=1\right\}, x^{\prime}=x /|x| \in \Sigma(x \neq 0)$, and $\rho$ any rotation of $\Sigma$ with magnitude $|\rho|=\sup _{x \in \Sigma}|\rho x-x|$. Let $1<r<\infty$ and $\Omega \in L^{r}(\Sigma)$ be positively homogeneous of degree zero. We say that $\Omega$ satisfies the $L^{r}$-Dini condition if

$$
\int_{0}^{1} \omega_{r}(\delta) \frac{d \delta}{\delta}<+\infty
$$

where

$$
\omega_{r}(\delta)=\sup _{|\rho|<\delta}\left(\int_{\Sigma}|\Omega(\rho x)-\Omega(x)|^{r} d \sigma_{x}\right)^{1 / r} .
$$

Set $K(x)=\Omega\left(x^{\prime}\right) /|x|^{n}$, with $\int_{\Sigma} \Omega(x) d \sigma_{x}=0$, and $T f(x)=(K * f)(x)$ in the usual principal-value sense. If $\Omega$ satisfies the $L^{r}$-Dini condition then it also satisfies the $L^{1}$-Dini condition, which by [1] implies $T$ is a bounded operator on $L^{p}, 1<p<\infty$. Recently, Kaneko and Yano [10] have shown that if $\Omega$ satisfies the $L^{\infty}$-Dini condition then $T$ maps $L_{w}^{p}$ into itself for $1<p<\infty$ and $w \in A_{p}$. We have extended this to:

TheOREM 4. Let $1<r<\infty, \Omega \in L^{r}(\Sigma)$, and $\int_{\Sigma} \Omega(x) d \sigma_{x}=0$. Suppose $\Omega$ satisfies the $L^{r}$-Dini condition. If

(1) $r^{\prime}<p<\infty$ and $w \in A_{p / r}$, or 
(2) $1<p<r$ and $w^{-1 /(p-1)} \in A_{p^{\prime} / r^{\prime}}$

then there is a constant $C$, independent of $f$, such that

$$
\|T f\|_{p, w}<C\|f\|_{p, w} \text {. }
$$

When $r<\infty$, we may take $p=r^{\prime}$ in (1) and $p=r$ in (2). If

(3) $w^{r^{\prime}} \in A_{1}$, then

$$
m_{w}\left(\left\{x \in \mathbf{R}^{n}:|T f(x)|>\lambda\right\}\right)<\frac{C}{\lambda}\|f\|_{1, w}, \quad \lambda>0,
$$

where $C$ is independent of $f$ and $\lambda$.

Theorem 4 is a direct analogue of Theorem 1. (We could also have stated a version of Theorem 3. See also [14].) In fact, when $r>2, r^{\prime}$ plays the same role as $n / l$. For example, notice the similarity between $m \in M(s, n), 1<s<$ 2 , and $\Omega$ satisfying the $L^{\infty}$-Dini condition. Our technique, however, does not allow for either $r$ or $s$ to be equal to 1 .

$\$ 2$ contains the basic lemma and a collection of results used in the proof of Theorem 1. This theorem and Theorems 2 and 3 are proved in \$3. The proof of Theorem 4 is found in $\$ 4$. The paper concludes with a counterexample showing Theorem 3 is best possible except for the question of endpoint equalities for $\beta$. The basic lemma and the counterexample are generalizations to $n>1$ of results in [16], and we gratefully acknowledge many helpful discussions with W.-S. Young and B. Muckenhoupt.

2. Following [7], we select an approximation to the identity

$$
\sum_{j=-\infty}^{+\infty} \phi\left(2^{-j} x\right)=1, \quad x \neq 0
$$

where $\varphi$ is an infinitely differentiable, nonnegative function supported in $\frac{1}{2}<|x|<2$. Let $m_{j}(x)=m(x) \varphi\left(2^{-j} x\right)$, so that

$$
m(x)=\sum_{j=-\infty}^{+\infty} m_{j}(x), \quad x \neq 0 .
$$

Notice that $m_{j}(x)$ is supported in $2^{j-1}<|x|<2^{j+1}$ and that for such $x$, $m_{k}(x)=0$ unless $k=j-1, j$, or $j+1$. It follows easily that if $m \in M(s, l)$ and $|\alpha|<l$, then

$$
\left(\int_{\mathbf{R}^{n}}\left|D^{\alpha} m_{j}(x)\right|^{s} d x\right)^{1 / s}<C\left(2^{j}\right)^{n / s-|\alpha|},
$$

with $C$ independent of $j$.

We also have that $m_{j} \in L^{1} \cap L^{\infty}$. Define $k_{j}(x)$ by $k_{j}(x)=\check{m}_{j}(x)$, and let

$$
m^{N}(x)=\sum_{j=-N}^{N} m_{j}(x), \quad K_{N}(x)=\left(m^{N}\right)^{\sim}(x)=\sum_{j=-N}^{N} k_{j}(x) .
$$

It follows that $\left\|m^{N}\right\|_{\infty}<C$, uniformly in $N$, and that $m^{N}(x) \rightarrow m(x), x \neq 0$, 
as $N \rightarrow \infty$. Now define $T_{N} f$ by $T_{N} f=\left(m^{N} \hat{f}\right)$, so that $T_{N} f=f * K_{N}$ for $f \in L^{2}$, say. The following lemma shows how conditions on $m$ can be interpreted as conditions on $K_{N}$.

LemMa 1. Let $1<s<2, m \in M(s, l)$ for a positive integer $l$, and let $K_{N}$ be defined as above. If $d$ is an integer such that $0<d<l, 1<t<s, n / t<d<$ $n / t+1$, and $1<p<t^{\prime}$, then

$$
\left(\int_{R<|x|<2 R}\left|K_{N}(x-y)-K_{N}(x)\right|^{p} d x\right)^{1 / p}<C R^{-d+n / p-n / r^{\prime}}|y|^{d-n / t}
$$

$$
\text { for all }|y|<\frac{R}{2},
$$

with $C$ independent of $N, R$, and $y$.

Proof. Since $K_{N}(x)=\sum_{j=-N}^{N} k_{j}(x)$,

$$
\begin{aligned}
\left(\int_{R<|x|<2 R}\left|K_{N}(x-y)-K_{N}(x)\right|^{p} d x\right)^{1 / p} & \\
& <\sum_{j}\left(\int_{R<|x|<2 R}\left|k_{j}(x-y)-k_{j}(x)\right|^{p} d x\right)^{1 / p} .
\end{aligned}
$$

Also, $|y|<R / 2$ and $R<|x|<2 R$ imply $R / 2<|x-y|<5 R / 2$, so that

$$
\begin{aligned}
\left(\int_{R<|x|<2 R}\left|k_{j}(x-y)-k_{j}(x)\right|^{p} d x\right)^{1 / p} & \\
& <\left(\int_{R<|x|<2 R}\left|k_{j}(x-y)\right|^{p} d x\right)^{1 / p}+\left(\int_{R<|x|<2 R}\left|k_{j}(x)\right|^{p} d x\right)^{1 / p} \\
& <2\left(\int_{R / 2<|x|<5 R / 2}\left|k_{j}(x)\right|^{p} d x\right)^{1 / p} .
\end{aligned}
$$

Therefore, we need to estimate

$$
\left(\int_{R / 2<|x|<5 R / 2}\left|k_{j}(x)\right|^{p} d x\right)^{1 / p} \text { and }\left(\int_{R<|x|<2 R}\left|k_{j}(x-y)-k_{j}(x)\right|^{p} d x\right)^{1 / p} \text {. }
$$

Let $d$ be an integer such that $0<d<l$ and $1<t<s$ such that $p<t^{\prime}$. It is easy to see that $m \in M(t, d)$. Let $x^{\alpha}=x_{1}^{\alpha_{1}} \cdots x_{n}^{\alpha_{n}}$. Then

$$
\begin{gathered}
\left(\int_{R / 2<|x|<5 R / 2}\left|k_{j}(x)\right|^{p} d x\right)^{1 / p}<C R^{-d}\left(\left.\left.\int_{R / 2<|x|<5 R / 2}|| x\right|^{d} k_{j}(x)\right|^{p} d x\right)^{1 / p} \\
<C R^{-d} \sum_{|\alpha|=d}\left(\int_{R / 2<|x|<5 R / 2}\left|x^{\alpha} k_{j}(x)\right|^{p} d x\right)^{1 / p} .
\end{gathered}
$$

Using the fact that $\check{m}_{j}=k_{j}$, Hölder's inequality, and the Hausdorff-Young 
theorem, we have for $|\alpha|=d$ that

$$
\begin{aligned}
\left(\int_{R / 2<|x|<5 R / 2}\left|x^{\alpha} k_{j}(x)\right|^{p}\right. & d x)^{1 / p}=\left(\int_{R / 2<|x|<5 R / 2}\left|\left(D^{\alpha} m_{j}\right)^{\nu}(x)\right|^{p} d x\right)^{1 / p} \\
& =R^{n / p}\left(R^{-n} \int_{R / 2<|x|<5 R / 2}\left|\left(D^{\alpha} m_{j}\right)^{\nu}(x)\right|^{p} d x\right)^{1 / p} \\
& <C R^{n / p}\left(R^{-n} \int_{R / 2<|x|<5 R / 2}\left|\left(D^{\alpha} m_{j}\right)^{-}(x)\right|^{t^{\prime}} d x\right)^{1 / t^{\prime}} \\
& <C R^{n / p-n / t^{\prime}}\left(\int_{R^{n}}\left|D^{\alpha_{m}}(x)\right|^{t} d x\right)^{1 / t} \\
& <C R^{n / p-n / t^{\prime}}\left(2^{j}\right)^{n / t-d} .
\end{aligned}
$$

Combining these estimates gives

$$
\left(\int_{R / 2<|x|<5 R / 2}\left|k_{j}(x)\right|^{p} d x\right)^{1 / p}<C R^{-d+n / p-n / t}\left(2^{j}\right)^{n / t-d} .
$$

For the integral of the difference of the $k_{j}$ 's we have

$$
\begin{aligned}
& \left(\int_{R<|x|<2 R}\left|k_{j}(x-y)-k_{j}(x)\right|^{p} d x\right)^{1 / p} \\
& =\left(\int_{R<|x|<2 R}\left|\left\{m_{j}(x)\left(e^{i x \cdot y}-1\right)\right\}\right|^{p} d x\right)^{1 / p} \\
& <C R^{-d}\left(\left.\int_{R<|x|<2 R}|| x\right|^{d}\left\{m_{j}(x)\left(e^{i x \cdot y}-1\right)\right\} y^{p} d x\right)^{1 / p} \\
& <C R^{n / p-d} \sum_{|\alpha|=d}\left(R^{-n} \int_{R<|x|<2 R}\left|x^{\alpha}\left\{m_{j}(x)\left(e^{i x \cdot y}-1\right)\right\}^{-p}\right|^{p} d x\right)^{1 / p} \\
& <C R^{n / p-d} \sum_{|\alpha|=d}\left(R^{-n} \int_{R<|x|<2 R}\left|\left\{D^{\alpha}\left[m_{j}(x)\left(e^{i x \cdot y}-1\right)\right]\right\}\right|^{p} d x\right)^{1 / p} \\
& <C R^{n / p-d} \sum_{|\alpha|=d}\left(R^{-n} \int_{R<|x|<2 R}\left|\left\{D^{\alpha}\left[m_{j}(x)\left(e^{i x \cdot y}-1\right)\right]\right\}\right|^{t^{\prime}} d x\right)^{1 / t^{\prime}} \\
& <C R^{n / p-d-n / t} \sum_{|\alpha|=d}\left(\int_{\mathbf{R}^{n}}\left|D^{\alpha}\left[m_{j}(x)\left(e^{i x \cdot y}-1\right)\right]\right|^{t} d x\right)^{1 / t} \\
& <C R^{n / p-d-n / t^{\prime}} \sum_{|\beta|+|\gamma|=d}\left(\int_{\mathbf{R}^{n}}\left|D^{\beta} m_{j}(x) \cdot D^{\gamma}\left(e^{i x \cdot y}-1\right)\right|^{t} d x\right)^{1 / t} .
\end{aligned}
$$


Consider first $|\gamma|=0,|\beta|=d$. Since $\left|e^{i x \cdot y}-1\right|<|x||y|$,

$$
\begin{aligned}
\left(\int_{\mathbf{R}^{n}}\left|\left(D^{\beta_{m_{j}}}(x)\right)\left(e^{i x \cdot y}-1\right)\right|^{t} d x\right)^{1 / t} & <\left(\int_{\mathbf{R}^{n}}|| x|| y\left|D^{\beta_{m_{j}}}(x)\right|^{t} d x\right)^{1 / t} \\
& <C 2^{j}|y|\left(2^{j}\right)^{n / t-d}=C|y|\left(2^{j}\right)^{n / t-d+1} .
\end{aligned}
$$

If $|\gamma|>0,\left|D^{\gamma}\left(e^{i x \cdot y}-1\right)\right|<|y|^{|\gamma|}$ and $|\beta|=d-|\gamma|$, so that

$$
\begin{aligned}
&\left(\int_{\mathbf{R}^{n}}\left|D^{\beta} m_{j}(x) \cdot D^{\gamma}\left(e^{i x \cdot y}-1\right)\right|^{t} d x\right)^{1 / t}<\left(\left.\left.\int_{\mathbf{R}^{n}}|| y\right|^{|\gamma|} D^{\beta} m_{j}(x)\right|^{t} d x\right)^{1 / t} \\
&<C|y|^{|\gamma|}\left(2^{j}\right)^{n / t-|\beta|}=C|y|^{|\gamma|}\left(2^{j}\right)^{n / t-d+|\gamma|} .
\end{aligned}
$$

Adding these estimates, we obtain

$\left(\int_{R<|x|<2 R}\left|k_{j}(x-y)-k_{j}(x)\right|^{p} d x\right)^{1 / p}<C R^{n / p-d-n / t^{\prime}} \sum_{m=1}^{d}|y|^{m}\left(2^{j}\right)^{n / t-d+m}$.

But, if $2^{j}<|y|^{-1}\left(|y|<2^{-j}\right)$,

$$
|y|^{m}\left(2^{j}\right)^{n / t-d+m}<|y|\left(2^{j}\right)^{n / t-d+1},
$$

so for these values of $j$, the estimate (2.3) becomes $C R^{n / p-d-n / t^{\prime}}|y|\left(2^{j}\right)^{n / t-d+1}$.

Using (2.2) and (2.3) in (2.1), we get

$$
\begin{aligned}
& \left(\int_{R<|x|<2 R}\left|K_{N}(x-y)-K_{N}(x)\right|^{p} d x\right)^{1 / p} \\
& \quad<C \sum_{2^{j}<|y|^{-1}} R^{n / p-d-n / t^{\prime}}|y|\left(2^{j}\right)^{n / t-d+1}+C \sum_{2^{j}>|y|^{-1}} R^{n / p-d-n / t^{\prime}}\left(2^{j}\right)^{n / t d} \\
& <C R^{n / p-d-n / t^{\prime}}|y|^{d-n / t}
\end{aligned}
$$

as long as $n / t<d<n / t+1$. This completes the proof of Lemma 1 .

Although we will not use it, we would like to point out that if $l>$ $\max \left\{n / p^{\prime}, n / s\right\}$, then

$$
\left(\int_{R<|x|<2 R}\left|K_{N}(x)\right|^{p} d x\right)^{1 / p}<C R^{n / p-n} .
$$

This follows from (2.2) with $d=l$ and the estimate

$$
\left(\int_{R<|x|<2 R}\left|k_{j}(x)\right|^{p} d x\right)^{1 / p}<C 2^{j n} R^{n / p},
$$

which is a consequence of $\left|k_{j}(x)\right|=\left|\check{m}_{j}(x)\right|<\left\|m_{j}\right\|_{1}<C 2^{j n}$.

REMARK 1. We may replace the domain of integration in Lemma 1 by $\left\{x \in \mathbf{R}^{n}: R<|x|\right\}$; that is, under the conditions of Lemma 1 , 


$$
\left(\int_{R<|x|}\left|K_{N}(x-y)-K_{N}(x)\right|^{p} d x\right)^{1 / p}<C R^{-d+n / p-n / t^{\prime}}|y|^{d-n / t} .
$$

For, if $t, d$, and $y$ satisfy the conditions of Lemma 1 ,

$$
\begin{aligned}
\left(\int_{R<|x|} \mid K_{N}\right. & \left.(x-y)-\left.K_{N}(x)\right|^{p} d x\right)^{1 / p} \\
& <\sum_{j=0}^{\infty}\left(\int_{2^{j} R<|x|<2^{j+1} R}\left|K_{N}(x-y)-K_{N}(x)\right|^{p} d x\right)^{1 / p} \\
& <\sum_{j=0}^{\infty} C\left(2^{j} R\right)^{-d+n / p-n / t^{\prime}}|y|^{d-n / t} \\
& =C R^{-d+n / p-n / t^{\prime}}|y|^{d-n / t} \sum_{j=0}^{\infty}\left(2^{j}\right)^{-d+n / p-n / t^{\prime}} \\
& =C R^{-d+n / p-n / t^{\prime}}|y|^{d-n / t},
\end{aligned}
$$

since $-d+n / p-n / t^{\prime}<0$ for $n / t<d$.

REMARK 2. The Hörmander-Mikhlin theorem follows easily from Lemma 1. To see this, let $m \in M(s, l), 1<s<2$ and $l>n / s$. Choose $t<s$ so that $n / t<l<n / t+1$. By Remark 1 with $p=1$ and $R=2|y|$, we have

$$
\int_{|x|>2|y|}\left|K_{N}(x-y)-K_{N}(x)\right| d x<C(2|y|)^{-l+n-n / t^{\prime}}|y|^{l-n / t}=C .
$$

Thus, the kernels $K_{N}$ satisfy, uniformly in $N$, the Hörmander condition

$$
\int_{|x|>2|y|}|K(x-y)-K(x)| d x<C \text { for all } y \neq 0,
$$

so that $T_{N} f=K_{N} * f$ is bounded on $L^{p}$, uniformly in $N$, for $1<p<\infty$.

For $f \in \mathcal{S}$, we have $T f=(m \hat{f})^{-}$. It follows that

$$
\left\|T f-T_{N} f\right\|_{\infty}<\left\|\left(m-m^{N}\right) \hat{f}\right\|_{1} \rightarrow 0
$$

since $m^{N}$ converges pointwise and boundedly to $m$. Then, applying Fatou's lemma, we get

$$
\|T f\|_{p}<C\|f\|_{p}
$$

for $f \in \mathcal{S}$, where $C$ is the uniform bound for the $T_{N}$ on $L^{p}$. The result extends to all of $L^{p}$ by continuity.

Part (1) of Theorem 1 is proved using Lemma 1 and the following three known results.

LEMMA 2. Set $f_{r}^{*}(x)=\left(\left(f^{r}\right)^{*}\right)^{1 / r}(x)$. If $0<r<p<\infty$ and $w \in A_{p / r}$, then

$$
\left\|f_{r}^{*}\right\|_{p, w}<C\|f\|_{p, w}
$$

with $C$ independent of $f$. 
This is an immediate corollary of results in [13].

LEMMA 3. Let

$$
f^{\sharp}(x)=\sup _{Q \ni x}|Q|^{-1} \int_{Q}\left|f(y)-\operatorname{av}_{Q} f\right| d y,
$$

where $\operatorname{av}_{Q} f=|Q|^{-1} \int_{Q} f(z) d z$. Let $0<p<\infty$ and $w \in A_{\infty}$. Then

$$
\left\|f^{*}\right\|_{p, w}<C\left\|f^{\sharp}\right\|_{p, w}
$$

with $C$ independent of $f$.

This is proved in [4]. The following result is a special case of interpolation with change of measures. It is proved in [17] and [19].

LEMMA 4. Let $1<r<q<\infty$ and let $w_{0}$ and $w_{1}$ be two positive weights. If $T$ is a bounded linear operator from $L_{w_{0}}^{r}$ into itself and $L_{w_{1}}^{q}$ into itself, then $T$ is bounded from $L_{w}^{p}$ into itself for $r<p<q$ and $w=w_{0}^{t} w_{1}^{1-t}$, provided $t=$ $(q-p) /(q-r)$ for $r \neq q$ and $0<t<1$ for $r=q$.

We would like to point out that $w^{n / l} \in A_{p}, n / l>1$, if and only if $w \in A_{p}$ and satisfies the reverse Hölder's inequalities

$$
\frac{1}{|Q|} \int_{Q} w^{n / l}(x) d x<C\left(\frac{1}{|Q|} \int_{Q} w(x) d x\right)^{n / l}
$$

and

$$
\frac{1}{|Q|} \int_{Q}\left(w(x)^{-1 /(p-1)}\right)^{n / l} d x<C\left(\frac{1}{|Q|} \int_{Q} w(x)^{-1 /(p-1)} d x\right)^{n / l} ;
$$

when $p=1$, we only need the first inequality. For $p>1$, if $w \in A_{p}$ and satisfies the above inequalities, then

$$
\begin{aligned}
\left(\frac{1}{|Q|} \int_{Q} w^{n / l}(x) d x\right) & \left(\frac{1}{|Q|} \int_{Q}\left(w^{n / l}(x)\right)^{-1 /(p-1)} d x\right)^{p-1} \\
& <C\left(\frac{1}{|Q|} \int_{Q} w(x) d x\right)^{n / l}\left(\frac{1}{|Q|} \int_{Q} w(x)^{-1 /(p-1)} d x\right)^{(p-1) n / l}
\end{aligned}
$$

so that $w^{n / l} \in A_{p}$. For $p=1$, if $w \in A_{1}$ and satisfies the first inequality above, then

$$
\begin{aligned}
\frac{1}{|Q|} \int_{Q} w^{n / l}(x) d x & <C\left(\frac{1}{|Q|} \int_{Q} w(x) d x\right)^{n / l} \\
& <C(\underset{Q}{\operatorname{essinf} w})^{n / l}=C \underset{Q}{\text { ess inf }} w^{n / l},
\end{aligned}
$$

so that $w^{n / l} \in A_{1}$. For the other implication, note first that $w^{n / l} \in A_{p}$ implies $w \in A_{p}$ since $n / l>1$. If $p>1$, by the $A_{p}$ condition, 


$$
\left(\frac{1}{|Q|} \int_{Q} w^{n / l}(x) d x\right)^{l / n} \leqslant C\left(\frac{1}{|Q|} \int_{Q} w(x)^{-(n / l)(1 /(p-1))} d x\right)^{-(l / n)(p-1)} .
$$

Thus, the first reverse Hölder's inequality will follow if we show

$$
\left(\frac{1}{|Q|} \int_{Q} w(x)^{-(n / l)(1 /(p-1))} d x\right)^{-(l / n)(p-1)}<\frac{1}{|Q|} \int_{Q} w(x) d x,
$$

or equivalently

$$
1<\left(\frac{1}{|Q|} \int_{Q} w(x) d x\right)\left(\frac{1}{|Q|} \int_{Q} w(x)^{-n / l(p-1)} d x\right)^{l(p-1) / n} .
$$

But, if $s>1$, using Hölder's inequality, we have

$$
\begin{aligned}
1 & =\frac{1}{|Q|} \int_{Q} d x=\frac{1}{|Q|} \int_{Q} w^{1 / s}(x) w^{-1 / s}(x) d x \\
& <\left(\frac{1}{|Q|} \int_{Q} w(x) d x\right)^{1 / s}\left(\frac{1}{|Q|} \int_{Q} w(x)^{-1 /(s-1)} d x\right)^{(s-1) / s} .
\end{aligned}
$$

Setting $s-1=l(p-1) / n$, or $s=1+l(p-1) / n>1$, we get the desired inequality. Since $w^{n / l} \in A_{p}$ implies $\left(w^{-1 /(p-1)}\right)^{n / l} \in A_{p^{\prime}}$, we also obtain the other reverse Hölder's inequality from the argument above. Finally, when $p=1$, by the $A_{1}$ condition

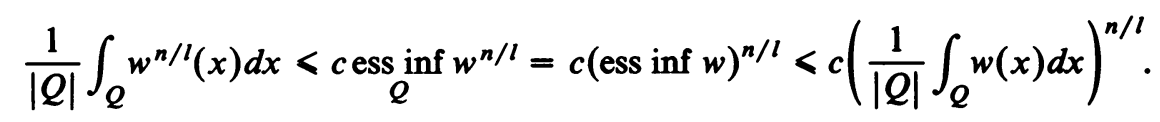

Notice that the above is true if we replace $n / l$ by any $t>1$.

3. We begin the proof of Theorem 1 by noting that (2) is a consequence of (1) by duality. To see this, suppose $1<p<(n / l)^{\prime}$ and $w^{-1 /(p-1)} \in A_{p^{\prime} l / n}$. Then, for $f \in \mathcal{S}$,

$$
\|T f\|_{p, w}=\left(\int_{\mathbf{R}^{n}}|T f(x)|^{p} w(x) d x\right)^{1 / p}=\sup \left|\int_{\mathbf{R}^{n}} T f(x) g(x) d x\right|,
$$

where the supremum is taken over all functions $g \in \mathcal{S}$ such that $\|g\|_{p^{\prime}, w^{-1 /(p-1)}}$ $=1$.

Let $\bar{T}$ be the operator with multiplier $\bar{m}$, the complex conjugate of $m$. Then $\bar{m}$ satisfies the same estimates as $m$ and we have

$$
\begin{aligned}
\|T f\|_{p, w} & =\sup \left|\int_{\mathbf{R}^{n}} f(x) \bar{T} g(x) d x\right|<\sup \|f\|_{p, w}\|\bar{T} g\|_{p^{\prime}, w^{-1 /(p-1)}} \\
& \leqslant C\|f\|_{p, w} \sup \|g\|_{p^{\prime}, w^{-1 /(p-1)}}=C\|f\|_{p, w}
\end{aligned}
$$

by (1), since $p^{\prime} \geq n / l$ and $w^{-1 /(p-1)} \in A_{p^{\prime} l / n}$.

Turning to the proof of (1), fix $p>n / l$ and $w \in A_{p l / n}$. Choose an $r<s$ such that $n / r$ is not an integer, $n / l<r<p$ and $w \in A_{p / r}$. There is an 
integer $d<l$ for which $n / r<d<n / r+1$. We will show

$$
\left(T_{N} f\right)^{\#}(x)<C f_{r}^{*}(x)
$$

with a $C$ independent of $f$ and $N$.

Fix $x \in \mathbf{R}^{n}$ and let $Q$ be a cube centered at $x$ with diameter $\delta$. Write

$$
f(y)=f_{0}(y)+\sum_{j=1}^{\infty} f_{j}(y)
$$

where

$$
f_{0}(y)=f(y) \chi\left(\left\{y \in \mathbf{R}^{n}:|x-y|<2 \delta\right\}\right)
$$

and

$$
f_{j}(y)=f(y) \chi\left(\left\{y \in \mathbf{R}^{n}: 2^{j} \delta<|x-y|<2^{j+1} \delta\right\}\right), \quad j=1,2, \ldots
$$

For $y \in Q$,

$$
\left(K_{N} * f\right)(y)=\left(K_{N} * f_{0}\right)(y)+\sum_{j=1}^{\infty}\left(K_{N} * f_{j}\right)(y) .
$$

By Hölder's inequality and Remark 2, for any $q>1$ we have

$$
\begin{aligned}
\frac{1}{|Q|} \int_{Q}\left|\left(K_{N} * f_{0}\right)(y)\right| d y & <\left(\frac{1}{|Q|} \int_{Q}\left|\left(K_{N} * f_{0}\right)(y)\right|^{q} d y\right)^{1 / q} \\
& <C \frac{\left\|f_{0}\right\|_{q}}{|Q|^{1 / q}}<C f_{q}^{*}(x),
\end{aligned}
$$

with $C$ independent of $N$. For any $j$,

$$
\begin{aligned}
\left(K_{N} * f_{j}\right)(y) & =\left(K_{N} * f_{j}\right)(x)+\int\left\{K_{N}(y-z)-K_{N}(x-z)\right\} f_{j}(z) d z \\
& \equiv c_{j}+\varepsilon_{j},
\end{aligned}
$$

say. Note that $c_{j}$ is independent of $y$ and

$$
\begin{aligned}
\left|\varepsilon_{j}\right|< & \int_{2^{j} \delta<|x-z|<2^{j+1} \delta}\left|K_{N}(y-z)-K_{N}(x-z)\right||f(z)| d z \\
< & \left(\int_{2^{j} \delta<|x-z|<2^{j+1 \delta}}\left|K_{N}(y-z)-K_{N}(x-z)\right|^{r^{\prime}} d z\right)^{1 / r^{\prime}} \\
& \cdot\left(\int_{|x-z|<2^{j+1 \delta}}|f(z)|^{r} d z\right)^{1 / r} .
\end{aligned}
$$

Applying Lemma 1 with $p=r^{\prime}$ and $t=r$ and noting that $|x-y|<\delta$, we obtain

$$
\begin{aligned}
\left|\varepsilon_{j}\right| & <C|x-y|^{d-n / r}\left(2^{j} \delta\right)^{-d}\left(2^{j+1} \delta\right)^{n / r}\left\{\left(2^{j+1} \delta\right)^{-n} \int_{|x-z|<2^{j+1} \delta}|f(z)|^{r} d z\right\}^{1 / r} \\
& <C\left(2^{j}\right)^{n / r-d} f_{r}^{*}(x) .
\end{aligned}
$$


Therefore,

$$
\begin{aligned}
\frac{1}{|Q|} \int_{Q \mid} \mid\left(K_{N} * f\right) & (y)-\sum_{j=1}^{\infty} c_{j}\left|d y=\frac{1}{|Q|} \int_{Q \mid}\right| \sum_{j=0}^{\infty}\left(K_{N} * f_{j}\right)(y)-\sum_{j=1}^{\infty} c_{j} \mid d y \\
& \leqslant \frac{1}{|Q|} \int_{Q}\left|\left(K_{N} * f_{0}\right)(y)\right| d y+\sum_{j=1}^{\infty} \frac{1}{|Q|} \int_{Q}\left|\left(K_{N} * f_{j}\right)(y)-c_{j}\right| d y \\
& \leqslant C f_{r}^{*}(x)+C \sum_{j=1}^{\infty}\left(2^{j}\right)^{n / r-d} f_{r}^{*}(x)=C f_{r}^{*}(x)
\end{aligned}
$$

since $n / r-d<0$. The fact that this estimate is true for any cube centered at $x$ implies (3.1). Now, using Lemmas 2 and 3, since $w \in A_{p / r}$, we obtain

$$
\left\|\left(K_{N} * f\right)\right\|_{p, w} \leqslant\left\|\left(K_{N} * f\right)^{*}\right\|_{p, w} \leqslant C\left\|\left(K_{N} * f\right)^{\sharp}\right\|_{p, w}<C\left\|f_{r}^{*}\right\|_{p, w}<C\|f\|_{p, w},
$$

uniformly in $N$. Arguing as in Remark 2, we have

$$
\|T f\|_{p, w}=\|(K * f)\|_{p, w}<C\|f\|_{p, w} .
$$

When $l<n$ and $p=n / l$, the above proof fails. However, using Lemma 4 and the fact that $w \in A_{1}$ implies there is a $b>1$ such that $w^{b} \in A_{1}$, we will prove the result. So, fix such a $b$. Then $w^{b} \in A_{q l / n}$ for any $q>n / l$. Setting $w_{0}(x)=1$ and $w_{1}(x)=w^{b}(x)$, we need to find $q$ and $r$ so that $r<n / l<q$ and $w(x)=\left(w^{b}(x)\right)^{(n / l-r) /(q-r)}$. Thus we need $b((n / l-r) /(q-r))=1$ or $b(n / l-r)=q-r$. Then, choosing $r, 1<r<n / l$, and solving for $q$, which is necessarily greater than $n / l$ since $b>1$, completes the proof.

The proof of Theorem 1 will be finished once we show the weak-type $(1,1)$ result. This will be done using standard techniques which are included for completeness. Fix a nonnegative $f$ in $L^{1} \cap L_{w}^{1}$ and $\lambda>0$. Applying the Calderón-Zygmund decomposition to $f$, we get a sequence of disjoint cubes $\left\{Q_{k}\right\}$ and functions $g$ and $b, f(x)=g(x)+b(x)$, satisfying

(i) $\left|Q_{k}\right|<(C / \lambda) \int_{Q_{k}} f(y) d y$,

(ii) $\|g\|_{2, w}^{2}<\lambda\|f\|_{1, w}$,

(iii) $b(y)=f(y)-\left|Q_{k}\right|^{-1} \int_{Q_{k}} f(z) d z$ for $y \in Q_{k}$, supp $b \subset \cup Q_{k}$ and $\int_{Q_{k}} b(y) d y=0$.

Since $T_{N} f=T_{N} g+T_{N} b$,

$$
\begin{aligned}
m_{w}(\{x & \left.\left.\in \mathbf{R}^{n}:\left|T_{N} f(x)\right|>2 \lambda\right\}\right) \\
& \leqslant m_{w}\left(\left\{x \in \mathbf{R}^{n}:\left|T_{N} g(x)\right|>\lambda\right\}\right)+m_{w}\left(\left\{x \in \mathbf{R}^{n}:\left|T_{N} b(x)\right|>\lambda\right\}\right) .
\end{aligned}
$$

We can apply (1) of Theorem 1 to the first term on the right because $w \in A_{1}$. Then, using (ii), we get

$$
m_{w}\left(\left\{x \in \mathbf{R}^{n}:\left|T_{N} g(x)\right|>\lambda\right\}\right)<\frac{C}{\lambda^{2}}\|g\|_{2, w}^{2}<\frac{C}{\lambda}\|f\|_{1, w} .
$$

Let $Q_{k}^{*}$ be $Q_{k}$ expanded concentrically twice. Then using (i) and the fact that 
$w \in A_{1}$, we have

$$
\begin{aligned}
m_{w}\left(\bigcup Q_{k}^{*}\right) & <\sum m_{w}\left(Q_{k}^{*}\right)<C \sum m_{w}\left(Q_{k}\right)<C \sum \frac{1}{\lambda} \int_{Q_{k}} f(y) \frac{m_{w}\left(Q_{k}\right)}{\left|Q_{k}\right|} d y \\
& <\frac{C}{\lambda} \sum \int_{Q_{k}} f(y) w(y) d y<\frac{C}{\lambda}\|f\|_{1, w} .
\end{aligned}
$$

Thus, we have only to show

$$
m_{w}\left(\left\{x \notin \bigcup Q_{k}^{*}:\left|T_{N} b(x)\right|>\lambda\right\}\right)<\frac{C}{\lambda}\|f\|_{1, w}
$$

Let $y_{k}$ and $\delta_{k}$ be the center and diameter of $Q_{k}$. Then

$$
\begin{aligned}
\int_{x \notin \cup Q_{k}^{*}}\left|T_{N} b(x)\right| w(x) d x=\int_{x \notin \cup Q_{k}^{*}}\left|\int_{\mathbf{R}^{n}} K_{N}(x-y) b(y) d y\right| w(x) d x \\
=\int_{x \notin \cup Q_{k}^{*}}\left|\sum_{k} \int_{Q_{k}} K_{N}(x-y) b(y) d y\right| w(x) d x \\
=\int_{x \notin \cup Q_{k}^{*}}\left|\sum_{k} \int_{Q_{k}}\left\{K_{N}(x-y)-K_{N}\left(x-y_{k}\right)\right\} b(y) d y\right| w(x) d x \\
\quad<\sum_{k} \int_{Q_{k}}\left(\int_{x \notin Q_{k}^{*}}\left|K_{N}(x-y)-K_{N}\left(x-y_{k}\right)\right| w(x) d x\right)|b(y)| d y .
\end{aligned}
$$

If we can show, for any $y \in Q_{k}$, that the inner integral is bounded by a constant independent of $k$ and $N$ times ess $\inf _{Q_{k}} w$, then our result will follow, as we now show. For, by (iii),

$$
\begin{aligned}
& m_{w}\left(\left\{x \notin \cup Q_{k}^{*}:\left|T_{N} b(x)\right|>\lambda\right\}\right)<\frac{1}{\lambda} \int_{x \notin \cup Q_{k}^{*}}\left|T_{N} b(x)\right| w(x) d x \\
& <\frac{C}{\lambda} \sum \int_{Q_{k}}|b(x)| \underset{Q_{k}}{\operatorname{ess} \inf } w d x<\frac{C}{\lambda} \sum \int_{Q_{k}}|b(x)| w(x) d x \\
& <\frac{C}{\lambda} \sum \int_{Q_{k}} f(x) w(x) d x+\frac{C}{\lambda} \sum \int_{Q_{k}}\left(\frac{1}{\left|Q_{k}\right|} \int_{Q_{k}} f(z) d z\right) w(x) d x \\
& <\frac{C}{\lambda}\|f\|_{1, w}+\frac{C}{\lambda} \sum \int_{Q_{k}} f(z) \frac{m_{w}\left(Q_{k}\right)}{\left|Q_{k}\right|} d z \\
& \leqslant \frac{C}{\lambda}\|f\|_{1, w}+\frac{C}{\lambda} \sum \int_{Q_{k}} f(z) w(z) d x<\frac{2 C}{\lambda}\|f\|_{1, w} \text {. }
\end{aligned}
$$

Therefore,

$$
m_{w}\left(\left\{x \in \mathbf{R}^{n}:\left|T_{N} f(x)\right|>\lambda\right\}\right)<\frac{C}{\lambda}\|f\|_{1, w}
$$

with a constant independent of $N, f$, and $\lambda$. If $f \in \mathcal{S}, f=f^{+}-f^{-}$where $f^{+}$ and $f^{-}$and nonnegative and in $L^{1} \cap L_{w}^{1}$, so that (3.3) holds for $f \in \mathcal{S}$. Then 


$$
\begin{aligned}
& m_{w}\left(\left\{x \in \mathbf{R}^{n}:|T f(x)|>\lambda\right\}\right) \\
&<m_{w}\left(\left\{x \in \mathbf{R}^{n}:\left|T_{N} f(x)\right|+\left|T f(x)-T_{N} f(x)\right|>\lambda\right\}\right) \\
&<m_{w}\left(\left\{x \in \mathbf{R}^{n}:\left|T_{N} f(x)\right|>\frac{\lambda}{2}\right\}\right) \\
&+m_{w}\left(\left\{x \in \mathbf{R}^{n}:\left|T f(x)-T_{N} f(x)\right|>\frac{\lambda}{2}\right\}\right) .
\end{aligned}
$$

Since $T_{N} f$ converges uniformly to $T f$ for $f \in \mathcal{S}$, choosing $N$ large enough the second term on the right is zero. By (3.3),

$$
m_{w}\left(\left\{x \in \mathbf{R}^{n}:|T f(x)|>\lambda\right\}\right)<\frac{C}{\lambda}\|f\|_{1, w} \text { for } f \in \mathcal{S},
$$

which extends to $L_{w}^{1}$.

To complete the proof of Theorem 1 we need to show

$$
\int_{x \notin Q_{k}^{*}}\left|K_{N}(x-y)-K_{N}\left(x-y_{k}\right)\right| w(x) d x<C \underset{Q_{k}}{\text { ess inf }} w \quad \text { if } y \in Q_{k},
$$

with $C$ independent of $k$ and $N$. Choose $r<s$ so that $n / r<l<n / r+1$ and $w^{r} \in A_{1}$. Then, using Lemma 1 with $p=r^{\prime}$ and $t=r$ and noting that $x \notin Q_{k}^{*}$ implies $\left|x-y_{k}\right|>2 \delta_{k}$, we have for $y \in Q_{k}$ that

$$
\begin{aligned}
& \int_{\left|x-y_{k}\right|>2 \delta_{k}}\left|K_{N}(x-y)-K_{N}\left(x-y_{k}\right)\right| w(x) d x \\
& =\sum_{j=1}^{\infty} \int_{2^{j} \delta_{k}<\left|x-y_{k}\right|<2^{j+1} \delta_{k}}\left|K_{N}(x-y)-K_{N}\left(x-y_{k}\right)\right| w(x) d x \\
& <\sum_{j=1}^{\infty}\left(\int_{2^{j} \delta_{k}<\left|x-y_{k}\right|<2^{j+1} \delta_{k}}\left|K_{N}(x-y)-K_{N}\left(x-y_{k}\right)\right|^{r} d x\right)^{1 / r} \\
& \cdot\left(\int_{\left|x-y_{k}\right|<2^{j+1} \delta_{k}} w^{r}(x) d x\right)^{1 / r} \\
& <C \sum_{j=1}^{\infty}\left(2^{j} \delta_{k}\right)^{-l}\left(\delta_{k}\right)^{l-n / r}\left(2^{j+1} \delta_{k}\right)^{n / r}\left\{\left(2^{j+1} \delta_{k}\right)^{-n} \int_{\left|x-y_{k}\right|<2^{j+1} \delta_{k}} w^{r}(x) d x\right\}^{1 / r} .
\end{aligned}
$$

Thus, since $w^{r} \in A_{1}$,

$$
\begin{aligned}
\int_{\left|x-y_{k}\right|>2 \delta_{k}} \mid & K_{N}(x-y)-K_{N}\left(x-y_{k}\right) \mid w(x) d x \\
& <C \sum_{j=1}^{\infty}\left(2^{j}\right)^{n / r-l} \underset{\left|x-y_{k}\right|<2^{j+1} \delta_{k}}{\operatorname{ess} \inf _{\mid}} w(x) \\
& <C \underset{\left|x-y_{k}\right|<\delta_{k}}{\operatorname{essinf}} w(x) \sum_{j=1}^{\infty}\left(2^{j}\right)^{n / r-l}<C \underset{Q_{k}}{\operatorname{ess} \inf } w
\end{aligned}
$$


with $C$ independent of $k$ and $N$. This completes the proof of Theorem 1.

We will derive Theorem 2 from Theorem 1 by using Lemma 4 and a characterization of $A_{p}$ functions proved by P. Jones [9]. He has shown that if $w \in A_{p}$ then there are $A_{1}$ weights $u$ and $v$ such that $w=v v^{1-p}$.

Fix $p, 1<p<\infty$, and $w$ so that $w^{n / l} \in A_{p}$. We have $w^{n / l}=u v^{1-p}$, $u, v \in A_{1}$, or $w=u^{l / n} v^{l(1-p) / n}$. Next, write this as

$$
w=u^{l / n} v^{l(1-p) / n}=\left(u^{\alpha} v^{\beta}\right)^{t}\left(u^{\gamma} v^{\delta}\right)^{1-t}=w_{0}^{t} w_{1}^{1-t}
$$

For this to make sense, we need

$$
\begin{gathered}
\alpha t+\gamma(1-t)=\frac{l}{n}, \\
\beta t+\delta(1-t)=\frac{l}{n}(1-p) .
\end{gathered}
$$

Then, in order to use Lemma 4 for weights which satisfy Theorem 1, we require

$$
\begin{gathered}
w_{0}^{-1 /(r-1)} \in A_{r l / n}, \quad 1<r<\min \left\{\left(\frac{n}{l}\right)^{\prime}, p\right\}, \\
w_{1} \in A_{q l / n}, \quad q>\max \left\{\frac{n}{l}, p\right\}, \\
t=\frac{q-p}{q-r} .
\end{gathered}
$$

Recall that $u \in A_{1}$ (similarly $v \in A_{1}$ ) implies

$$
\frac{1}{|Q|} \int_{Q} u(y) d y<C u(x) \text { for almost all } x \in Q \text {. }
$$

Therefore, if $\alpha>0$ and $\beta<0$, letting $s=r^{\prime} l / n$, we have

$$
\begin{aligned}
&\left(\frac{1}{|Q|} \int_{Q} w_{0}(x)^{-1 /(r-1)} d x\right)\left(\frac{1}{|Q|} \int_{Q} w_{0}(x)^{(1 /(r-1))(1 /(s-1))} d x\right)^{s-1} \\
&=\left(\frac{1}{|Q|} \int_{Q} u(x)^{-\alpha /(r-1)} v(x)^{-\beta /(r-1)} d x\right) \\
& \cdot\left(\frac{1}{|Q|} \int_{Q} u(x)^{(\alpha /(r-1))(1 /(s-1))} v(x)^{(\beta /(r-1))(1 /(s-1))} d x\right)^{s-1} \\
&< C\left(\frac{1}{|Q|} \int_{Q} u(x) d x\right)^{-\alpha /(r-1)}\left(\frac{1}{|Q|} \int_{Q} v(x)^{-\beta /(r-1)} d x\right) \\
& \cdot\left(\frac{1}{|Q|} \int_{Q} v(x) d x\right)^{\beta /(r-1)}\left(\frac{1}{|Q|} \int_{Q} u(x)^{(\alpha /(r-1))(1 /(s-1))} d x\right)^{s-1} \\
&= C,
\end{aligned}
$$


if

$$
\alpha=(r-1)\left(\frac{r^{\prime} l}{n}-1\right)=\frac{r l}{n}-r+1 \text { and } \beta=-(r-1) ;
$$

that is $w_{0}^{-1 /(r-1)} \in A_{r l / n}$ for these values of $\alpha$ and $\beta$. Similarly, we can show $w_{1} \in A_{q l / n}$ if $\gamma=1$ and $\delta=-((q l / n)-1)$. Using these values of $\alpha$ and $\gamma$, we have (3.4) if $t=1 / r$. Next, solving (3.5) for $q$, we get $q=r^{\prime}(p-1)$. This value of $q$ also satisfies (3.8). Therefore, if we choose $r<\min \left\{(n / l)^{\prime}, p\right\}$ so close to 1 that $q=r^{\prime}(p-1)>\max \{n / l, p\}$, we can satisfy (3.4)-(3.8), proving Theorem 2 .

Before proving Theorem 3, notice that $-n>-l p$ if $n / l<p$, and $n(p-1)<l p$ if $p \leqslant(n / l)^{\prime}$. Therefore, for $l<n$ the conclusion of Theorem 3 can be divided into three cases:

$$
\begin{gathered}
1<p<\frac{n}{l} \text { and }-l p<\beta<n(p-1), \\
\frac{n}{l}<p<\left(\frac{n}{l}\right)^{\prime} \text { and }-n<\beta<n(p-1), \\
\left(\frac{n}{l}\right)^{\prime}<p<\infty \text { and }-n<\beta<l p .
\end{gathered}
$$

Since (3.11) is the dual of (3.9), we need only concern ourselves with (3.9) and (3.10).

Next, let us interpret Theorem 1 when $w(x)$ is a power of $|x|$. Because $|x|^{\beta} \in A_{p}$ if and only if $-n<\beta<n(p-1)$, we have $(l<n)$ that $T$ is bounded on $L p_{\left.x\right|^{\beta}}$ if

$$
\begin{gathered}
\quad \frac{n}{l} \leqslant p<\infty \text { and }-n<\beta<p l-n, \\
1<p \leqslant\left(\frac{n}{l}\right)^{\prime} \text { and }-n+p(n-l)<\beta<n(p-1) .
\end{gathered}
$$

However, combining (3.12) and (3.13), we have (3.10) and are left with only proving (3.9).

Let $q=n / l$ and $r<n / l$; then also $r<(n / l)^{\prime}$. By (3.13) and (3.10), $T$ is bounded on $L_{|x|^{\beta_{0}}}^{r}$ and $L_{|x|^{\beta_{1}}}^{q}$ for $-n+r(n-l)<\beta_{0}<n(r-1)$ and $-n<$ $\beta_{1}<n(q-1)$. Using Lemma 4 , if $r<p<q$ we see that $T$ is bounded on $L_{|x|^{\beta}}$ for

$$
\beta=\beta_{0}\left(\frac{q-p}{q-r}\right)+\beta_{1}\left(\frac{p-r}{q-r}\right)
$$

Thus $\beta$ satisfies

$$
\begin{aligned}
\{-n+r(n-l)\} & \left(\frac{q-p}{q-r}\right)-n\left(\frac{p-r}{q-r}\right) \\
<\beta & <n(r-1)\left(\frac{q-p}{q-r}\right)+n(q-1)\left(\frac{p-r}{q-r}\right) .
\end{aligned}
$$


Simplifying and using the fact that $q=n / l$, we get

$$
\frac{n^{2}(r-1)}{n-l r}+\frac{p l r(l-n)}{n-l r}<\beta<n(p-1)
$$

But, as $r \rightarrow 1$, the left-hand side of (3.14) approaches - lp. So, taking $r$ sufficiently close to 1 allows us to choose any $\beta$ satisfying $-l p<\beta<$ $n(p-1)$.

When $l=n$, the restriction in Theorem 3 is $-n<\beta<n(p-1)$ for $1<p<\infty$. But, when $l=n$ in Theorem 1 , we require $w \in A_{p}$, and $|x|^{\beta} \in$ $A_{p}$ if $-n<\beta<n(p-1)$.

4. The proof of Theorem 4 is based on an analogue of Lemma 1.

LEMMA 5. Let $\Omega \in L^{r}(\Sigma)$ and satisfy the $L^{r}$-Dini condition. Set $K(x)=$ $\Omega\left(x^{\prime}\right) /|x|^{n}$. There exists a constant $\alpha_{0}>0$ such that if $|y|<\alpha_{0} R$, then

$$
\begin{aligned}
\left(\int_{R<|x|<2 R}|K(x-y)-K(x)|^{r} d x\right)^{1 / r} & \\
& <C R^{n / r-n}\left\{\frac{|y|}{R}+\int_{|y| / 2 R<\delta<|y| / R} \omega_{r}(\delta) \frac{d \delta}{\delta}\right\} .
\end{aligned}
$$

Proof. We may choose $\alpha_{0}<\frac{1}{2}$; then, since $|x|>R,|x-y|$ is equivalent to $|x|$. Therefore,

$$
\begin{aligned}
|K(x-y)-K(x)| & =\left|\frac{\Omega(x-y)}{|x-y|^{n}}-\frac{\Omega(x)}{|x|^{n}}\right| \\
& <C\left\{|\Omega(x)| \frac{|y|}{|x|^{n+1}}+\frac{|\Omega(x-y)-\Omega(x)|}{|x|^{n}}\right\} .
\end{aligned}
$$

It follows that

$$
\begin{aligned}
\left(\int_{R<|x|<2 R}|K(x-y)-K(x)|^{r} d x\right)^{1 / r} \\
<C\left(\int_{R<|x|<2 R}|\Omega(x)|^{r} \frac{|y|^{r}}{|x|^{(n+1) r}} d x\right)^{1 / r} \\
+C\left(\int_{R<|x|<2 R} \frac{|\Omega(x-y)-\Omega(x)|^{r}}{|x|^{n r}} d x\right)^{1 / r} .
\end{aligned}
$$

The first term on the right side of (4.1) is bounded by

$$
C\|\Omega\|_{L^{\prime}(\Sigma)}|y| R^{-(n+1)} R^{n / r}=C R^{n / r-n}\left(\frac{|y|}{R}\right) .
$$


Changing to polar coordinates, we see the second term equals

$$
\begin{aligned}
C\left(\int_{R}^{2 R} t^{-n r+n-1}\left(\int_{\Sigma}\left|\Omega\left(t x^{\prime}-y\right)-\Omega\left(t x^{\prime}\right)\right|^{r} d \sigma_{x^{\prime}}\right) d t\right)^{1 / r} \\
<C R^{n / r-n}\left(\int_{R}^{2 R}\left(\int_{\Sigma}\left|\Omega\left(\frac{x^{\prime}-\alpha}{\left|x^{\prime}-\alpha\right|}\right)-\Omega\left(x^{\prime}\right)\right|^{r} d \sigma_{x^{\prime}}\right) \frac{d t}{t}\right)^{1 / r},
\end{aligned}
$$

where $\alpha=y / t$. Arguing as in Calderón, Weiss, and Zygmund [1, pp. 65-72], we see the inner integral is bounded by

$$
C \sup _{|\rho|<|\alpha|} \int_{\Sigma}\left|\Omega\left(\rho x^{\prime}\right)-\Omega\left(x^{\prime}\right)\right|^{r} d \sigma_{x^{\prime}}=C \omega_{r}^{r}\left(\frac{|y|}{t}\right)
$$

as long as $|\alpha|=|y| / t<\alpha_{0}$. Thus, the second term is bounded by

$$
\begin{aligned}
C R^{n / r-n}\left(\int_{R}^{2 R} \omega_{r}^{r}\left(\frac{|y|}{t}\right) \frac{d t}{t}\right)^{1 / r} & =C R^{n / r-n}\left(\int_{|y| / 2 R}^{|y| / R} \omega_{r}^{r}(\delta) \frac{d \delta}{\delta}\right)^{1 / r} \\
& <C R^{n / r-n}\left(\int_{|y| / 2 R<\delta<|y| / R} \omega_{r}(\delta) \frac{d \delta}{\delta}\right),
\end{aligned}
$$

since $\omega_{r}$ is essentially constant on intervals of the form $(a, 2 a), a>0$. Lemma 5 is now proved.

Notice that when $R=2^{j}|y|$, with a $j$ such that $1 / \alpha_{0}<2^{j}$, we get

$$
\begin{aligned}
\left(\int_{2^{j}|y|<|x|<2^{j+1}|y|}|K(x-y)-K(x)|^{r} d x\right)^{1 / r} \\
<C\left(2^{j}|y|\right)^{n / r-n}\left\{\frac{1}{2^{j}}+\int_{2^{-(j+1)}}^{2^{-j}} \omega_{r}(\delta) \frac{d \delta}{\delta}\right\} .
\end{aligned}
$$

Theorem 4 is proved in exactly the same manner as Theorem 1. Using Lemma 5, we show

$$
(K * f)^{\sharp}(x)<C f_{r}^{*}(x),
$$

which proves the result for $p>r^{\prime}$. The only change necessary is in the decomposition $f=f_{0}+\sum f_{j}$. For Theorem 4,

$$
f_{0}(y)=f(y) \chi\left(\left\{y \in \mathbf{R}^{n}:|x-y|<\frac{1}{\alpha_{0}} \delta\right\}\right)
$$

and the sum of $f_{j}^{\prime}$ 's is over $j>\log _{2}\left(1 / \alpha_{0}\right)$. We get the case $p=r^{\prime}$ by interpolation, and $1<p<r$ follows by duality. In the weak-type $(1,1)$ proof, we may have to replace the weak-type $(2,2)$ result for the good function by a weak-type $\left(r^{\prime}, r^{\prime}\right)$ result.

5. We conclude by showing that Theorem 3 is best possible, except for endpoint equalities for $\beta$. We prove the result for $p>(n / l)^{\prime}$; the case $p<n / l$ follows by duality. For $n / l<p<(n / l)^{\prime}$, the Riesz transforms and 
an argument like that in [8] show the range of $\beta$ is best possible.

Let $1<s<2, n / s<l<n,(n / l)^{i}<p$ and $\beta>l p$. Define a multiplier $m$ by

$$
m(x)=e^{i x \cdot \eta}\left(1+|x|^{2}\right)^{-l / 2}
$$

for a fixed $\eta$ of length 1 . Note that $\check{m}(x)=G_{l}(x-\eta)$ (the Bessel kernel of order $l)$ and that $\left|D^{\alpha} m(x)\right|<C_{\alpha} /(1+|x|)^{l}$, so $m \in M(s, l), 1<s<\infty$. Moreover, $G_{l}>0$ and there exist $c, \mu>0$ such that $G_{l}(x)>c|x|^{l-n}$ if $|x|<\mu$ (see Stein [19, p. 132] for details).

Set

$$
f(x)=|x|^{-((n+\beta) / p)}|\log | x||^{-\delta} \chi\left(\left\{x \in \mathbf{R}^{n}:|x|<\mu\right\}\right) .
$$

If $\delta p>1, f \in L_{|x|^{\beta}}^{p}\left(\mathbf{R}^{n}\right)$. Since $T f(x)=\left(G_{l}(\cdot-\eta) * f\right)(x)$,

$$
\begin{aligned}
T f(x) & =\int_{|y|<\mu}|y|^{-((n+\beta) / p)}|\log | y||^{-\delta} G_{l}(x-y-\eta) d y \\
& =\int_{|x-\eta-z|<\mu}|x-\eta-z|^{-((n+\beta) / p)}|\log | x-\eta-z||^{-\delta} G_{l}(z) d z
\end{aligned}
$$

by setting $z=x-\eta-y$. Now, if we restrict the integration to $|z|<$ $\frac{1}{2}|x-\eta|,|x-\eta-z|$ is equivalent to $|x-\eta|$ and, if $|x-\eta|<\mu / 2$,

$$
\begin{aligned}
T f(x) & \geqslant C|x-\eta|^{-((n+\beta) / p)}|\log | x-\eta||^{-\delta} \int_{|z|<\frac{1}{2}|x-\eta|} \frac{d z}{|z|^{n-l}} \\
& =C|x-\eta|^{l-((n+\beta) / p)}|\log | x-\left.\eta\right|^{-\delta} .
\end{aligned}
$$

Therefore, $T f \notin L_{|x|^{\beta}}^{p}\left(\mathbf{R}^{n}\right)$ if $\{l-((n+\beta) / p)\} p<-n$; i.e., if $\beta>l p$.

\section{REFERENCES}

1. A. P. Calderón, M. Weiss and A. Zygmund, On the existence of singular integrals, Proc. Sympos. Pure Math., vol. 10, Amer. Math. Soc., Providence, R. I., 1967, pp. 56-73.

2. A. P. Calderón and A. Torchinsky, Parabolic maximal functions associated with a distribution. II, Advances in Math. 24 (1977), 101-171.

3. R. Coifman and C. Fefferman, Weighted norm inequalities for maximal functions and singular integrals, Studia Math. 51 (1974), 241-250.

4. A. Cordóba and C. Fefferman, $A$ weighted norm inequality for singular integrals, Studia Math. 57 (1976), 97-101.

5. C. Fefferman and E. M. Stein, $H^{p}$ spaces of several variables, Acta Math. 129 (1972), 137-193.

6. I. I. Hirschman, Jr., The decomposition of Walsh and Fourier series, Mem. Amer. Math. Soc., no. 15, Amer. Math. Soc., Providence, R. I., 1955.

7. L. Hörmander, Estimates for translation invariant operators in $L^{p}$ spaces, Acta Math. 104 (1960), 93-139.

8. R. Hunt, B. Muckenhoupt and R. Wheeden, Weighted norm inequalities for the conjugate function and the Hilbert transform, Trans. Amer. Math. Soc. 176 (1973), 227-251.

9. P. Jones, (to appear). 
10. M. Kaneko and S. Yano, Weighted norm inequalities for singular integrals, J. Math. Soc. Japan 27 (1975), 570-588.

11. P. Krée, Sur les multiplicateurs dans FLP avec poids, Ann. Inst. Fourier (Grenoble) 16 (1966), 91-121.

12. D. Kurtz, Littlewood-Paley and multiplier theorems on weighted $L^{P}$ spaces, Ph.D. Dissertation, Rutgers University, 1978.

13. B. Muckenhoupt, Weighted norm inequalities for the Hardy maximal function, Trans. Amer. Math. Soc. 165 (1972), 207-226.

14. B. Muckenhoupt and $\mathbf{R}$. Wheeden, Weighted norm inequalities for singular and fractional integrals, Trans. Amer. Math. Soc. 161 (1971), 249-258.

15. , Norm inequalities for the Littlewood-Paley function $\mathbf{g}_{\lambda}^{*}$, Trans. Amer. Math. Soc. 191 (1974), 95-111.

16. B. Muckenhoupt, R. Wheeden and W.-S. Young, (to appear).

17. E. Stein, Interpolation of linear operators, Trans. Amer. Math. Soc. 83 (1956), 482-492.

18. Singular integrals and differentiability properties of functions, Princeton Univ. Press, Princeton, N. J., 1970.

19. E. Stein and G. Weiss, Interpolation of operators with change of measures, Trans. Amer. Math. Soc. 87 (1958), 159-172.

20. H. Triebel, Spaces of distributions with weights, multipliers in $L^{P}$-spaces with weights, Math. Nachr. 78 (1977), 339-355.

Department of Mathematics, Purdue University, West LafayetTe, Indiana 47907

Department of Mathematics, Rutgers University, New Brunswick, New Jersey 08903 